\title{
On Algebras Associated with Integrable Hamiltonian Systems
}

\author{
Stanisław Kasperczuk \\ University of Zielona Góra, Institute of Physics, ul. Szafrana 4a, 65-516 Zielona Góra, Poland \\ e-mail: S.Kasperczuk@if.uz.zgora.pl
}

(Received: 23 March 2010; accepted: 8 July 2010; published online: 13 September 2010)

\begin{abstract}
The aim of this paper is to give a general setting, based on quantum deformations, for the explicit construction of certain classes of integrable Hamiltonian systems.
\end{abstract}

Key words: Poisson bialgebras, Casimir functions, Hamiltonian systems, integrability, quantum deformations

\section{INTRODUCTION}

Integrable Hamiltonian systems play a fundamental role in the study and description of physical systems, due to their many interesting properties, both from the mathematical and physical points of view. Indeed, beyond the obvious interest of finding first integrals, the concept of integrability seems necessary for more thorough understanding of the nonintegrability phenomenon. Integrable Hamiltonian systems always have a hidden algebraic structure that is responsible for their integrability. Therefore, the most interesting problem in the study of dynamical systems is to give such a general algebraic structure which provides a hidden treasure. To date, however, there exists no general method for determining whether or not a given system is integrable. Even in the simplest nontrivial case, i.e. in twodegree of freedom Hamiltonian systems, our knowledge is far from the desired goal. In recent years there has been a renewed interest in completely integrable Hamiltonian systems, especially in conjunction with the study of quantum integrable systems and quantum groups.

This paper presents a procedure in order to construct complete integrable Hamiltonian systems with arbitrary many degrees of freedom from a Poisson bialgebra $\left(\mathcal{F}(\mathcal{L})_{\alpha}, P, \Delta, \varepsilon,\{,\}_{F}\right)$ on symplectic leaves $\mathcal{L}_{\alpha}$ of a Poisson manifold $\left(\mathbb{R}^{3}, P\right)$. This construction was put into a geometrical perspective in Refs. [1-3]. We modify the algebraic structure of a Poisson bialgebras by considering the deformed coproduct and deformations of bialgebras. These modifications lead to the quantum groups and provide new classes of completely integrable Hamiltonian systems.

\section{POISSON BIALGEBRAS AND INTEGRABLE SYSTEMS}

First let us recall some algebraic preliminaries. Detailed exposition can be found for example in Refs. [4-6]. A unital associative algebras over $K$ is a linear space $A$ together with two linear maps $m: A \otimes A \rightarrow A$ and $\eta ; K \rightarrow A$ so that: $m(m \otimes \mathbf{1})=m(\mathbf{1} \otimes m)$, and $m(\mathbf{1} \otimes \eta)=m(\eta \otimes \mathbf{1})=i d$. Let $\left(A_{1}, m_{1}, \eta_{1}\right)$ and $\left(A_{2}, m_{2}, \eta_{2}\right)$ be algebras, then the tensor product $A_{1} \otimes A_{2}$ is naturally endowed with the structure of an algebra. The multiplication $m_{a_{1} \otimes A_{2}}$ is defined by:

$$
\left(a_{1} \otimes a_{2}\right)\left(b_{1} \otimes b_{2}\right)=\left(a_{1} b_{1}\right) \otimes\left(a_{2} b_{2}\right) .
$$

A coalgebra is a triple $(A, \Delta, \mathcal{E})$ with a linear space $A$ over $K, \Delta: A \rightarrow A \otimes A$ a linear map called comultiplication and $\mathcal{E}: A \rightarrow K$ a linear morphism called counit with property

(i) $\Delta(a b)=\Delta(a) \Delta(b), \forall a, b \in A$,

(ii) $(\Delta \otimes i d) \circ \Delta=(i d \otimes) \circ \Delta$,

(iii) $($ id $\otimes \varepsilon) \circ \Delta=(\varepsilon \otimes i d) \circ \Delta=i d$.

We note that $A \otimes A$ is both an algebra and a coalgebra of $A$. The space of smooth functions $\mathcal{F}(N)$ on a symplectic submanifold $N$ is a Poisson algebra. A tensor product of Poisson algebras $\mathcal{F}(N) \otimes \mathcal{F}(N)$ is again a Poisson algebra. We have to define a Poisson structure on $\mathcal{F}(N) \otimes \mathcal{F}(N)$ such that the axioms of Poisson algebra are satisfied. For our purpose the maps are defined as follows. The multiplication $m_{\mathcal{F} \otimes \mathcal{F}}$

$$
\Delta\left(x_{i}\right)=x_{i} \otimes \mathbf{1}+\mathbf{1} \otimes x_{i} .
$$

The Poisson structure on $\mathcal{F}(N)$

$$
\{f, g\}_{\mathcal{F}}=\left\{x_{a}, x_{b}\right\} \frac{\partial f}{\partial x_{a}} \frac{\partial g}{\partial x_{b}} .
$$


We define the following Poisson bracket on $\mathcal{F}(N) \otimes \mathcal{F}(N)$ :

$$
\{f \otimes g, h \otimes j\}_{\mathcal{F} \otimes \mathcal{F}}=\{f, g\}_{\mathcal{F}} \otimes g j+f h \otimes\{g, j\}_{\mathcal{F}} \cdot(3)
$$

We will say that the set $\left(\mathcal{F}(N), m, \Delta, \mathcal{E},\{,\}_{\mathcal{F}}\right)$ is a Poisson bialgebra. Assume $\mathcal{C}$ is a Casimir for the Poisson manifold $\left(\mathbb{R}^{\mathbf{3}}, P\right)$, then for any $h \in \mathcal{F}\left(\mathbb{R}^{\mathbf{3}}\right)$ and $N_{\alpha}=\mathcal{C}^{-1}(\alpha)$, $\alpha \in \mathbb{R}$

$$
\{\mathcal{C}, h\} \mid N_{\alpha}=\{\tilde{\mathcal{C}}, \tilde{h}\}_{\mathcal{F}}=0
$$

The above relation defines an integrable Hamiltonian system with two degrees of freedom, given by any function $\widetilde{h} \in \mathcal{F}(N)$ and with the second integral generated by the Casimir $\mathcal{C}$.

\section{II.1. Bianchi's algebra}

In this subsection we write an explicit formula for the Poisson bialgebras structure arising from the Poisson-Lie structure. We start with the Lie algebra $e(2)$ [1]: $\left[e_{1}, e_{2}\right]=e_{3}, \quad\left[e_{2}, e_{3}\right]=0,\left[e_{3}, e_{1}\right]=e_{2}$. This solvable algebra is of the type $\mathrm{VII}_{0}$ Bianchi's classification and is isomorphic to the Euklidean algebra of the plane. We consider the dual $e(2)^{*}$ to $e(2)$ equipped with the linear Poisson-Lie structure

$$
P=x_{3} \partial_{1} \wedge \partial_{2}+x_{2} \partial_{3} \wedge \partial_{1} .
$$

Poisson bracket in the space of smooth functions on $e(2)^{*}$ is defined according to the formula: $\{F, G\}=P(d F, d G)$, $F, G \in \mathcal{F}\left(e\left(2^{*}\right)\right)$. A Casimir of $P$ is

$$
C=x_{2}^{2}+x_{3}^{2}
$$

and $e(2)^{*}$ decomposes into a foliation by symplectic submanifolds $N_{r}=C^{-1}(r), r \in \mathbb{R}$. Since the level sets of $C$ are circular cylinders, we choose the usual cylinder coordinates: $x_{1}=p, x_{2}=r \sin q, x_{3}=r \cos q, r=\sqrt{C}$. The transformation of the basis in the tangent space is: $\partial_{1}=\partial_{p}$, $\partial_{2}=\sin q \partial_{r}+r^{-1} \cos q \partial_{q}, \partial_{3}=\cos q \partial_{r}-r^{-1} \sin q \partial_{q}$. Let $h: N_{r} \rightarrow \mathbb{R}$ be any smooth function with $H \mid N_{r}=h$. If $f=F \mid N_{r}$, the Poisson bracket $\{f, h\}$ is defined by restriction $\{F, H\}$ to $N_{r}$. The Poisson structure $P_{a b} \mid N_{r}$ is defined by (5). In terms of coordinates $(p, q)$ on $N_{r}$ the Poisson bracket of $f$ and $h$ reads (see e.g. [7])

$$
\{f, h\}=\{p, q\}_{P}\left(\frac{\partial f}{\partial p} \frac{\partial h}{\partial q}-\frac{\partial f}{\partial q} \frac{\partial h}{\partial p}\right)
$$

An easy calculation shows that $\{p, q\}_{P}=1$, hence $p, q$ are canonical coordinates on $N_{r}$ (cf. [1]). From (6) we obtain: $x_{1}=p, x_{2}=\sin q, x_{3}=\cos q$. Setting:

$$
f(p, q) \otimes \mathbf{1}=f\left(p_{1}, q_{1}\right),
$$

$$
\begin{gathered}
\mathbf{1} \otimes f(p, q)=f\left(p_{2}, q_{2}\right), \\
f(p, q) \otimes h(p, q)=f\left(p_{1}, q_{1}\right) h\left(p_{2}, q_{2}\right)
\end{gathered}
$$

we get

$$
\Delta(\mathrm{c})=1+\cos \left(q_{2}-q_{1}\right)
$$

Thus any Hamiltonian system $\left(\mathbb{R}^{4}, \omega, H\right)$ with Hamiltonian $H\left(p_{1}, p_{2}, q_{1}, q_{2}\right)=\Delta(h(p, \sin q, \cos q))$ is completely integrable if $d H \wedge d(\Delta(\mathrm{c})) \neq 0$ where $\omega^{-1}=\{\} \times 1+,1 \times\{$,$\} .$

\section{MODIFIED ALGEBRAIC STRUCTURES}

\section{III.1. Deformed coproduct}

Let us first introduce the deformed coproduct

$$
\begin{aligned}
& \tilde{\Delta}(x)=x \otimes \mathbf{1}+\mathbf{1} \otimes x, \quad \tilde{\Delta}(y)=y \otimes a_{1}+a_{2} \otimes y, \\
& \tilde{\Delta}(z)=z \otimes a_{1}+a_{2} \otimes z, \quad \lim _{\epsilon \rightarrow 0}\left(a_{i}(x, \epsilon)\right)=1 .
\end{aligned}
$$

According to our procedure in Sec. II we have

$$
\begin{gathered}
\tilde{\Delta}(x)=p_{1}+p_{2}, \quad \tilde{\Delta}(y)+e^{\epsilon p_{2}} \sin q_{1}=e^{-\epsilon p_{1}} \cos q_{2}, \\
\tilde{\Delta}(z)=e^{\epsilon p_{2}} \cos q_{1}+e^{-\epsilon p_{1}} \cos q_{2} .
\end{gathered}
$$

The deformed coproduct of the Casimir

$$
\tilde{\Delta}(c)=e^{-2 \epsilon p_{1}}+e^{2 \epsilon p_{2}}+2 e^{\epsilon\left(p_{2}-p_{1}\right)} \cos \left(q_{2}-q_{1}\right),
$$

defines again a family of integrable Hamiltonian systems.

\section{III.2. Deformed algebra structure}

Now we introduce the deformed Poisson tensor

$$
\tilde{P}=\tilde{z} \partial_{\tilde{x}} \wedge \partial_{\tilde{y}}+\tilde{y} \partial_{\tilde{z}} \wedge \partial_{\tilde{x}}+g(x, \epsilon) \partial_{\tilde{y}} \wedge \partial_{\tilde{z}},
$$

where $\tilde{x}=x, \quad \tilde{y}=f(x, \epsilon), \quad \lim _{\epsilon \rightarrow 0} g=0, \lim _{\epsilon \rightarrow 0} f=1$. A Poisson coalgebra structure is obtained by means of the coproduct

$$
\begin{gathered}
\tilde{\Delta}(\tilde{x})=\tilde{x} \otimes \mathbf{1}+\mathbf{1} \otimes \tilde{x}, \quad \tilde{\Delta}(\tilde{y})=\tilde{y} \otimes e^{\epsilon \tilde{x} / 2}+e^{-\epsilon \tilde{x} / 2} \otimes \tilde{y}, \\
\tilde{\Delta}(\tilde{z})=\tilde{z} \otimes e^{\epsilon \tilde{x} / 2}+e^{-\epsilon \tilde{x} / 2} \otimes \tilde{z} .
\end{gathered}
$$

A relation that satisfies all the requirements is $\tilde{x}=p, \tilde{y}=2 \cosh (\epsilon p / 2) \sin q, \tilde{z}=2 \cosh (\epsilon p / 2) \cos q$. (14)

Hence

$$
\begin{aligned}
& \tilde{\Delta}(\tilde{c})=e^{\epsilon p_{2}} \cosh ^{2}\left(\epsilon p_{1} / 2\right)+e^{-\epsilon p_{1} / 2} \cosh ^{2}\left(\epsilon p_{1} / 2\right)+ \\
& +2 \cosh \left(\epsilon p_{1} / 2\right) \cosh \left(\epsilon p_{2} / 2\right) e^{\epsilon\left(p_{2}-p_{1}\right)} \cos \left(q_{2}-q_{1}\right)
\end{aligned}
$$




\section{CONCLUSIONS}

1. The procedure to obtain integrable Hamiltonian systems with two degrees of freedom can be generalized to any number of degrees of freedom by making use of the $k$-th coproduct. Letting $\Delta=\Delta_{1}$ we find $\Delta_{k+1}=\left(\Delta \otimes i d^{k}\right) \circ \Delta_{k}$, that is, diagonalizing on the first factor after applying $\Delta_{k}$. Hence, for arbitrary $k \geq 2$, we have

$$
\begin{gathered}
\Delta_{k-1}(\mu)=\sum_{i=1}^{k} \mu_{i}, \\
\left\{\Delta_{k-1}(\mu), \Delta_{k-1}(\eta)\right\}^{k}=\sum_{i=1}\{\mu, \eta\}_{i},
\end{gathered}
$$

where $\mu, \eta$ are linear coordinates on $e(2)^{*}$. The integrals of a Hamiltonian system with $n$ degrees of freedom are given by $n-1$ coproducts of the Casimir $F_{i}=\Delta_{i}(c), i=1,2, \ldots, n-1$, and arbitrary Hamiltonian $H=\Delta_{n-1}(h(\tilde{x}, \tilde{y}, \tilde{z}))$. An easy computation shows that $H, F_{1}, \ldots, F_{n-1}$ are in involution, and $d H \wedge d F_{i} \neq 0$, $d F_{i} \wedge d F_{j} \neq 0, i \neq j$.

2. The coproduct (9):

$$
\begin{gathered}
\tilde{\Delta}(x)=x \otimes \mathbf{1}+\mathbf{1} \otimes x, \tilde{\Delta}(y)=y \otimes e^{\epsilon x}+e^{-\epsilon x} \otimes y, \\
\tilde{\Delta}(z)=z \otimes e^{\epsilon x}+e^{-\epsilon x} \otimes z
\end{gathered}
$$

defines the quantum deformation $U_{\epsilon}(e(2))$ (cf. $\left.[5,6]\right)$.
3. The deformed structure (12) of $e(2):\left[\widetilde{e}_{1}, \widetilde{e}_{1}\right]=\widetilde{e}_{3}$, $\left[\tilde{e}_{3}, \tilde{e}_{1}\right]=\tilde{e}_{2},\left[\tilde{e}_{2}, \tilde{e}_{1}\right]=-\epsilon \sinh \left(\epsilon \tilde{e}_{1}\right)$ with the deformed coproduct (13):

$$
\begin{gathered}
\tilde{\Delta}\left(\tilde{e}_{1}\right)=\tilde{e}_{1} \otimes \mathbf{1}+\mathbf{1} \otimes \tilde{e}_{1}, \\
\tilde{\Delta}\left(\tilde{e}_{2}\right)=\tilde{e}_{2} \otimes e^{\epsilon \tilde{e}_{1} / 2}+e^{-\epsilon \tilde{e}_{1} / 2} \otimes e_{2}, \\
\tilde{\Delta}\left(\tilde{e}_{3}\right)=\tilde{e}_{3} \otimes e^{\epsilon \tilde{e}_{1} / 2}+e^{-\epsilon \tilde{e}_{1} / 2} \otimes \tilde{e}_{3},
\end{gathered}
$$

may be identified with the nonstandard quantum deformation of algebra $e(2)$ (cf. [5, 6]).

\section{References}

[1] S. Kasperczuk, Cel. Mech. Dyn. Astron. 76, 215 (2000).

[2] S. Kasperczuk, Physics A 284, 113 (2000).

[3] S. Kasperczuk, Acta Physica Polonica B 34, 11 (2003).

[4] M.A.E. Sweedler, Hopf Algebras, Reading. MA: AdisonWesley, New York 1969.

[5] S. Maijd, Foundations of Quantum Groups Theory. Camberidge Univ. Press 1995.

[6] Chari, A. Pressley, A Guide to Quantum Groups. Camberidge Univ. Press 2000.

[7] A. Weinstein, J. Diff. Geom. 18, 523 (1983).

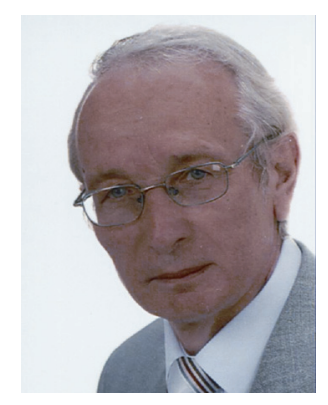

DSc. Stanislaw Kasperczuk is Associate Professor at the Department of Physics and Astronomy of the University of Zielona Góra. His research field is the study of dynamical systems. 